\title{
Implementation of a patient data management system in an ICU
}

Marcel van Vliet RN, BN Senior Nurse, Adult Intensive Care, Academic Medical Centre, Amsterdam, The Netherlands

E-mail: m.w.vanvliet@amc.uva.nl

Key words: information technology intensive care unit patient data management system (PDMS)

\section{SUMMARY}

* The aim of this paper is to describe the process of acquisition and implementation of a patient data management system (PDMS) into an intensive care unit (ICU).

* A PDMS can provide support in nursing care and may prove to be a valuable instrument in improving the quality of ICU care.

* To be successful, the implementation process requires careful planning in order to be accepted by the medical and nursing staff.

\section{INTRODUCTION}

The Academic Medical Centre in Amsterdam is one of the largest teaching hospitals in The Netherlands, with 1,000 beds and 7,000 employees. One of its goals is to explore new developments in both medical and nursing fields. The Centre has recently implemented a new patient data management system (PDMS) in its intensive care unit (ICU), which has 27 beds providing care for all categories of medical and surgical adult patients. Since April 2002, 16 of these beds have been equipped with a 100MBPS (megabytes per second) network: ICU LAN (local area network), bedside stations, and a PDMS. The introduction of a PDMS necessitates communication with a variety of commercially-available medical devices present at the bedside. The PDMS is diagrammatically presented in Figure 1. Based on our experience, this paper describes the process of implementing the PDMS.

\section{What is a PDMS?}

A PDMS is an electronic dossier supporting the user in patient care (Weiss \& Sprung, 1996). A variety of user-programmed data can be collected electronically (see examples in Figure 2). In addition, a PDMS can provide management information and is a useful tool for research purposes. It is a computer-based information system to facilitate the collection, integration, retrieval and interpretation of the multi-source, multi-variant data found in intensive care units (Milholland, 1995). Limited information is available in the literature concerning the implementation of a PDMS.

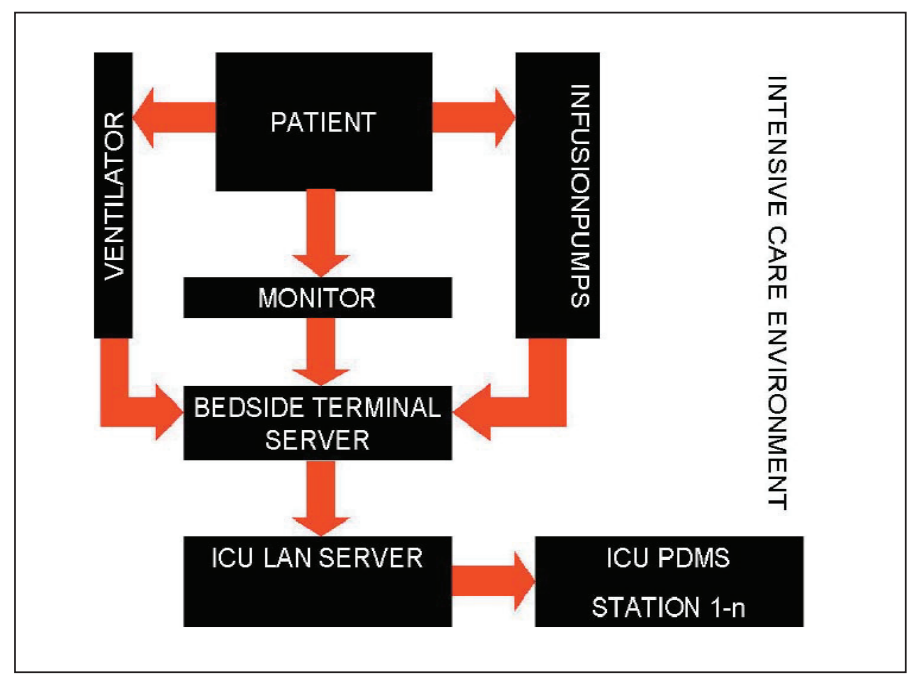

Figure 1. The ICU environment of the PDMS.

\section{Benefits to nursing care}

The field of intensive care nursing is one of continuously advancing technology. The number of bedside devices continues to increase and includes technologies such as ventilators, vital-signs monitors and, in more recent years, haemofiltration equipment, intra-aortal balloon pump devices and intra-cranial pressure monitoring systems. The attending nurse is frequently confronted with many sources of information that lack an integrated structure. Traditionally, data produced by the various monitors are recorded manually on large paper charts, usually kept at the bedside. A PDMS can process all the generated data more easily (Metnitz \& Lenz, 1995). Moreover, the PDMS reduces time spent on administrative nursing tasks, thereby increasing the amount of time available for direct patient care. For example, in our unit, the number of prescribed drugs per patient has increased over time. Drug prescription lists are also manually recorded and copied each night to provide a complete overview for the next day. The copying process, as a result, is subject to human error. The same applies for the manual recording of laboratory results and pharmacy orders. A system that can eliminate the need for manual copying 


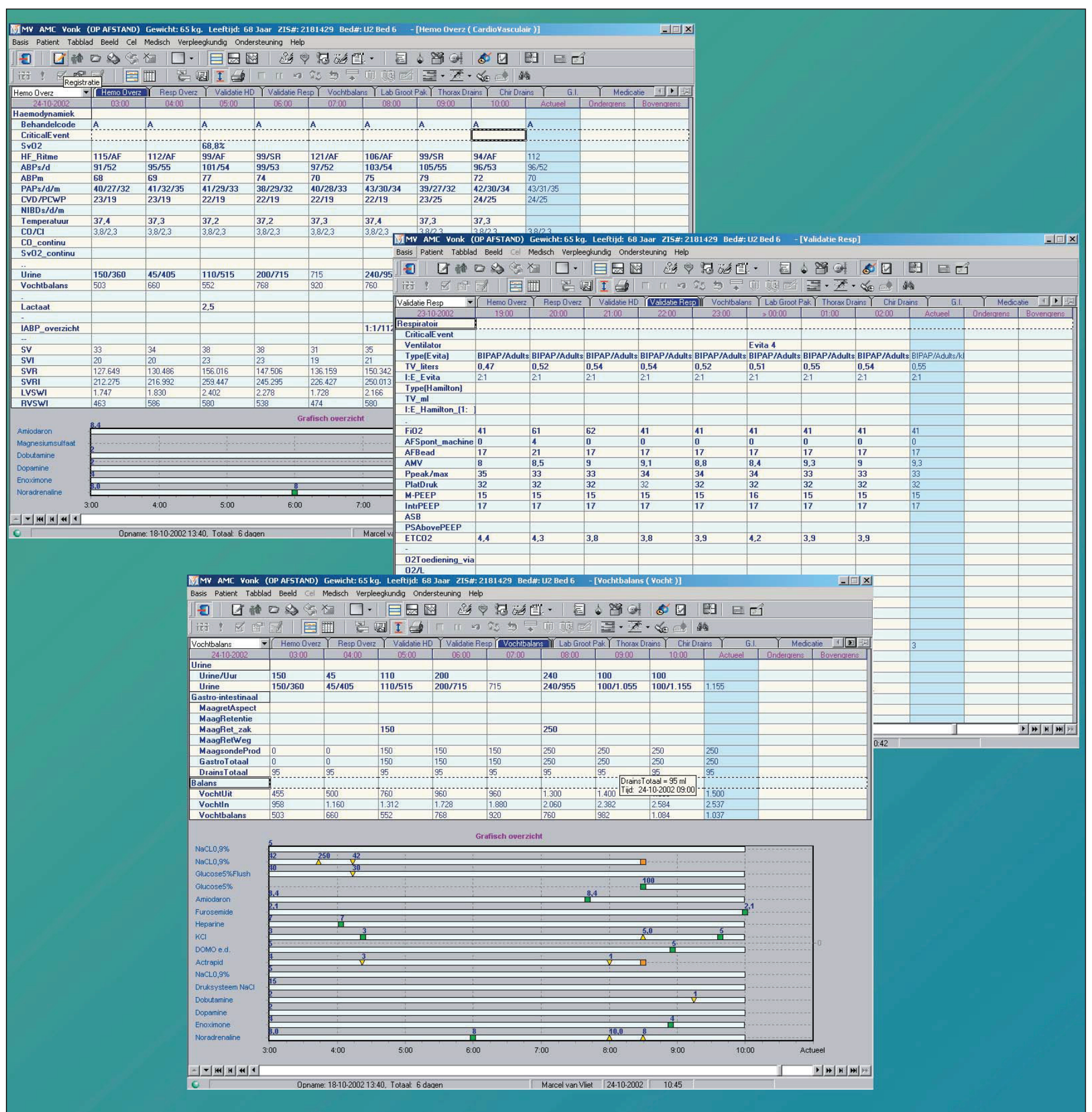

Figure 2. PDMS data screens.

of medications and other vital orders can be integrated into the PDMS, thus ensuring all available patient information is accessible from any workstation (see Figure 3). Only data authorised by the bridge device can go to the ICU LAN server. Figure 3 shows how the ICU LAN interacts with the different departments in our hospital. Besides administrative support, the PDMS also provides nurses with bedside access to technical support, protocols and procedures, as well as a drug compendium.

Previously, in our intensive care unit, APACHE (Acute Physiology and Chronic Health Evaluation) and other scoring systems were also recorded by hand. The handwritten sheets hampered the quality of data collection. For instance, the lowest arterial blood pressure would be recorded as the lowest value noted on the paper charts, whereas the nurse at the bedside may not have recorded the patient's actual lowest value. Since there is a great need for reliable data for medical and nursing analyses, the addition of a data management tool can enhance the possibilities for medical and nursing research. Finally, the PDMS may prove to be 
a valuable adjunct in the assessment of the costs of treatment per patient.

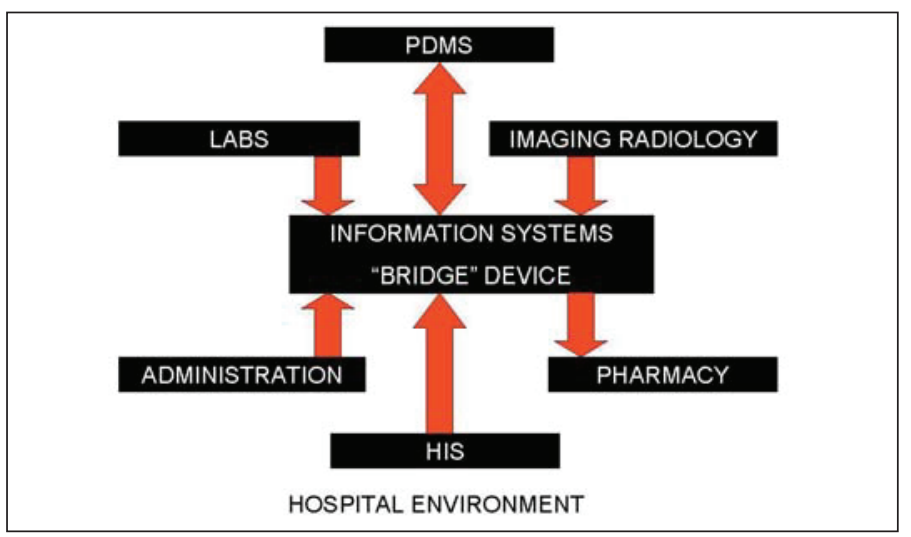

Figure 3. Hospital environment of the PDMS.

\section{IMPLEMENTATION OF THE PDMS}

An interest group was formed to define the requirements of a PDMS. The group consisted of information technology (IT) specialists, and intensive care unit nursing and medical staff. The group identified the desired functions of the PDMS as:

* Patient registration and administration, allowing transfer of data and correspondence to third parties;

* Observation and monitoring - registration of all data output from monitoring devices;

* Registration of all medications;

* Provision of online support for nurses and doctors (for example treatment and care plans);

* Acquisition of management information about groups of patients;

- Provision of a database for scientific research about groups of patients;

* Ensuring privacy and security.

Secondary demands involved cost management tools, stock management, registration of machine availability and network access tools (linking beds to nurses/doctors/units). Evaluation of older systems (De Keizer et al, 1998) prior to 2002 revealed significant technical and configuration problems. Patient data management systems have improved since then, and the current Visual Basic type applications and easy access to the underlying database meet most requirements. The Academic Medical Centre selected the iMDsoft software from Metavision.

Implementing a PDMS is a challenging task that requires major financial support (Norrie \& Blackwell, 2000). Renovation of our ICU environment was necessary to create the infrastructure for bedside workstations, and LAN and server rooms that met the standards for conversion to digital format. The IT specialists designed the infrastructure, and nurses and doctors conducted education programmes for the staff.

During the implementation phase, a 'super-users' group and the system administrators were appointed. Their functions were to:

* Configure the system and layout to be acceptable for ICU implementation;

* Educate their co-workers, by building an educational programme;

* Ensure data quality by active screening and education;
* Prioritise needs for the system;

* Warrant data quality after implementation by supporting their co-workers on the work floor;

* Develop configuration and applications after implementation;

* Communicate with back-end services. (See Figure 4).

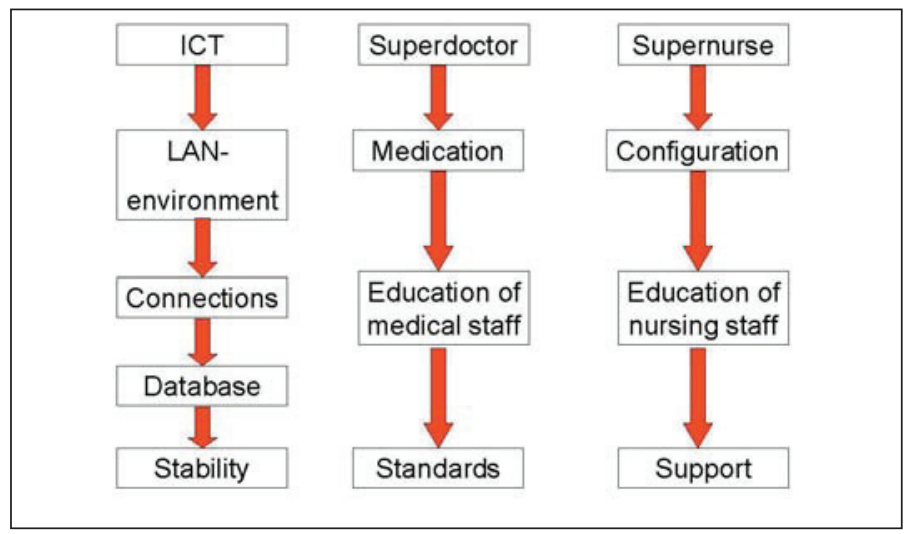

Figure 4. Different tasks for different professions.

To educate approximately 100 ICU staff members, a series of one-day group training sessions was organised, with small groups of up to four people led by one or two trainers (super-users, see Figure 4). The entire ICU staff was educated within five weeks. Doctors and nurses received different education programmes, as their interactions with a PDMS are different. The nurses' emphasis was on validating data and managing fluids, whereas the doctors' emphasis was mainly on giving orders and maintaining an overview. Following the education programmes, the system went 'live' in the ICU. Super-users were present 24 hours a day to offer support to users and to check data integrity until all staff had adjusted to the new PDMS. Errors were recorded in an incident logbook and were used later for evaluation and development purposes.

\section{Acceptance by staff}

Besides the quality of the system, the success of a specific information system is determined largely by the level of acceptance by the users. In our experience, staff acceptance was influenced strongly by investment in both system configuration (the extent to which the product meets the users' demands) and education.

Several key factors influenced acceptance:

* The high level of support given by super-users;

* The involvement of users in the levels of configuration. For example, layout, standards for medication;

* The level of education and training provided;

* The degree of staff satisfaction with increased time avail able for patient-related care as opposed to administrative tasks.

The workgroup realised that implementing a new system in the intensive care unit was an important change, and that implementation should be gradual rather than sudden. Each step in the process was carefully planned and communicated to staff by paper-based and intranet communications, and educational programmes.

\section{CONCLUSION}

Implementing a PDMS in an intensive care unit is a challenging 
task. It requires high input of human and financial resources. Careful evaluation of requirements is needed for successful implementation. Education is the key to creating a platform from which nurses and doctors can work. Support provided on the work floor, by specially trained staff, increases both general acceptance and improves the integrity of data management. With the increasing number of bedside machines and data provided by them, the PDMS is a promising administrative tool for accounting and processing that data.

\section{REFERENCES}

De Keizer NF, Stoutenbeek CP, Hanneman LA \& De Jonge E.

(1998) An evaluation of Patient Data Management Systems in
Dutch intensive care. Intensive Care Medicine 24 (2): 167171.

Metnitz PG \& Lenz K. (1995) Patient data management systems in intensive care - the situation in Europe. Intensive Care Medicine 21 (9): 703-715.

Milholland DK. (1995) Information systems in critical care: a measure of their effectiveness. Medinfo 8 (2): 1068-1070.

Norrie P \& Blackwell RE. (2000) Constructing a financial case for a computerized patient data management system (PDMS) in a cardiothoracic intensive care unit. Medical Informatics and the Internet in Medicine 25 (3): 161-169.

Weiss Y \& Sprung C. (1996) Patient data management systems in critical care. Current Opinion in Critical Care 2: 187-192. 